\title{
Ausblick: Rehabilitation - quo vadis?
}

\author{
Ulf Seifart für den Arbeitskreis Onkologische Rehabilitation in der DGHO
}

Klinik Sonnenblick, Marburg, Deutschland

Während die ambulante und die stationäre Behandlung onkologischer Patienten umfänglich akzeptiert sind, wird der Sektor der onkologischen Rehabilitation teilweise sehr kritisch diskutiert.

So wird der Rehabilitation insgesamt und auch speziell der onkologischen Rehabilitation vorgeworfen, dass die angebotenen Therapien wenig evidenzbasiert und wenig nachhaltig sind. Diese Einwände sind im Prinzip durchaus berechtigt, allerdings weisen aktuelle Datenerhebungen in eine andere Richtung:

Dies ist unter anderem in der sozialmedizinischen Kompetenz der Rehabilitation begründet. Sie bietet den Patienten eine der wenigen Möglichkeiten, sich über die Vielfalt der Hilfsangebote zu informieren und sich bezüglich ihrer beruflichen oder sozialen Wiedereingliederung zu orientieren.

Standen in der Onkologie bislang Zielkriterien wie die Verlängerung des Gesamtüberlebens oder des progressionsfreien Überlebens im Fokus des Interesses, gewinnt in jüngster Zeit der Erhalt von Lebensqualität an Bedeutung. In diesem Sinne ist auch die Durchführung einer onkologischen Reha-Maßnahme zur Erhaltung von Lebensqualität und Autonomie und damit einhergehend zur Vermeidung oder Verschiebung von Pflegebedürftigkeit eine geeignete Maßnahme. An ausgewählten Aspekten der onkologischen Rehabilitation lässt sich dies veranschaulichen:

\section{Sport und Krebs}

Trotz methodischer Schwierigkeiten gibt es zwischenzeitlich eine relativ große Zahl an Studien zu nichtmedikamentösen Ansätzen in der onkologischen Rehabilitation. Besonders gut untersucht ist die Wirksamkeit der Sport- und Bewegungstherapie, deren zahlreiche positive Auswirkungen gut belegt und evidenzgestützt sind. Zusammenfassend hat sich in großen Metaanalysen gezeigt, dass sich bei Probanden mit regelmäßiger körperlicher Bewegung die aerobe Fitness bessert, Körpergewicht und Körperfett abnehmen und die Muskelkraft zunimmt. Darüber hinaus ließ sich eine Vielzahl günstiger psychosozialer Effekte nachweisen. Bei einigen Tumorentitäten (z.B. Mammakarzinom, Kolonkarzinom, Prostatakarzinom) scheint körperliche Aktivität auch mit einer Verringerung der Rezidiv- und Mortalitätsrate einherzugehen.

\section{Psychoonkologie}

Der Nutzen psychoonkologischer Angebote in der Rehabilitation wird ebenfalls von einer ausreichend großen Datenlage gestützt, wobei Ernährungsempfehlungen und Programme zur medizinisch-beruflich orientierten Rehabilitation (MBOR) diesbezüglich noch weiter geprüft werden sollten. Auch wurde der für Deutschland spezifische Ansatz eines «multimodalen Reha-Settings» bisher kaum in Studien berücksichtigt. Zudem sind die überwiegend untersuchten Einzelinterventionen äußerst heterogen, was die Vergleichbarkeit erschwert. Die unter Reha-Aspekten am besten untersuchte Tumorentität ist das Mammakarzinom. Für andere Tumoren sind zunächst weitere Studienergebnisse abzuwarten.

\section{Return to Work}

Neben den oben genannten Aspekten übernimmt die Sozialmedizin eine wichtige Rolle im Hinblick auf die Lebensqualität, insbesondere der langzeitüberlebenden Krebspatienten. Derzeit sind etwa 700000 Tumorpatienten mit der Frage nach dem «Return to work» konfrontiert. Nach aktuellen Studien- und Registerdaten ist davon auszugehen, dass nur etwa die Hälfte dieser Patienten wieder voll berufstätig wird. Dies geht zum Teil mit erheblichen finanziellen Belastungen und Einschnitten für die Patienten einher. In einer bis dato nicht publizierten Untersuchung konnte gezeigt werden, dass allogen transplantierte Patienten im Durchschnitt 520 Euro ihres monatlichen Einkommens verlieren. Zudem drohen unseren Patienten bei Verlust des Arbeitsplatzes erhebliche finanzielle Belastungen. So erhält ein Patient mit einem monatlichen Durchschnittseinkommen von 2126 Euro ein Arbeitslosengeld I von 841 Euro pro Monat, sofern keine Kinder zu versorgen sind. Bei Bezug einer Erwerbsminderungsrente wird bei gleichen finanziellen Voraussetzungen ein monatlicher Betrag von 683,57 Euro ausgezahlt.

Um den drohenden sozialen Abstieg zu vermeiden, bietet unser Sozialsystem eine Fülle an Hilfsangeboten, deren Auswahl und Beantragung aber zeitintensiv sind und Kenntnisse in der Sozialmedizin verlangen. Aus diesem Grund benötigen unsere Patienten Hilfestellungen bzw. einen Lotsen, der sie durch den «Paragraphen-Dschungel» leitet. In Zeiten reduzierter Sozialdienste an den Krankenhäusern und fraglicher Finanzierung der Krebsberatungsstellen der Landeskrebsgesellschaften stellt die Reha-Einrichtung hier einen möglichen Ansprechpartner dar.

\section{Fazit}

Abschließend darf festgestellt werden, dass die moderne Rehabilitation einen wichtigen Beitrag in der gesamtheitlichen Betreuung onkologischer Patienten leistet. Diskussionswürdig sind weiterhin das unübersichtliche Zuweisungsverfahren und die Vorgaben bezüglich einer starren Verweildauer der Patienten. Hier wird es notwendig sein, eine Flexibilisierung und Vereinfachung der Verfahren zu erreichen.

\section{KARGER}

Fax +497614520714 